\title{
Statistical analysis of the dependence of large-scale Birkeland currents on solar wind parameters
}

\author{
H. Korth ${ }^{1}$, B. J. Anderson ${ }^{1}$, and C. L. Waters ${ }^{2}$ \\ ${ }^{1}$ The Johns Hopkins University, Applied Physics Laboratory, Laurel, MD, USA \\ ${ }^{2}$ School of Mathematical and Physical Sciences, The University of Newcastle, NSW, Australia
}

Received: 24 September 2009 - Revised: 15 January 2010 - Accepted: 25 January 2010 - Published: 10 February 2010

\begin{abstract}
The spatial distributions of large-scale fieldaligned Birkeland currents have been derived using magnetic field data obtained from the Iridium constellation of satellites from February 1999 to December 2007. From this database, we selected intervals that had at least $45 \%$ overlap in the large-scale currents between successive hours. The consistency in the current distributions is taken to indicate stability of the large-scale magnetosphere-ionosphere system to within the spatial and temporal resolution of the Iridium observations. The resulting data set of about 1500 two-hour intervals ( $4 \%$ of the data) was sorted first by the interplanetary magnetic field (IMF) GSM clock angle $\left(\arctan \left(B_{\mathrm{y}} / B_{\mathrm{z}}\right)\right)$ since this governs the spatial morphology of the currents. The Birkeland current densities were then corrected for variations in EUV-produced ionospheric conductance by normalizing the current densities to those occurring for $0^{\circ}$ dipole tilt. To determine the dependence of the currents on other solar wind variables for a given IMF clock angle, the data were then sorted sequentially by the following parameters: the solar wind electric field in the plane normal to the Earth-Sun line, $E_{\mathrm{yz}}$; the solar wind ram pressure; and the solar wind Alfvén Mach number. The solar wind electric field is the dominant factor determining the Birkeland current intensities. The currents shift toward noon and expand equatorward with increasing solar wind electric field. The total current increases by $0.8 \mathrm{MA}$ per $\mathrm{mV} \mathrm{m}^{-1}$ increase in $E_{\mathrm{yz}}$ for southward IMF, while for northward IMF it is nearly independent of the electric field, increasing by only $0.1 \mathrm{MA}$ per $\mathrm{mV} \mathrm{m}^{-1}$ increase in $E_{\mathrm{yz}}$. The dependence on solar wind pressure is comparatively modest. After correcting for the solar dynamo dependencies in intensity and distribution, the total current intensity increases with solar wind dynamic pressure by $0.4 \mathrm{MA} / \mathrm{nPa}$ for southward IMF. Normalizing the Birke-
\end{abstract}

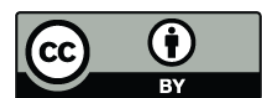

Correspondence to: $\mathrm{H}$. Korth

(haje.korth@jhuapl.edu) land current densities to both the median solar wind electric field and dynamic pressure effects, we find no significant dependence of the Birkeland currents on solar wind Alfvén Mach number.

Keywords. Ionosphere (Auroral ionosphere; Electric fields and currents; Ionosphere-magnetosphere interactions)

\section{Introduction}

Field-aligned Birkeland currents play a fundamental role in conveying stresses in the coupled solar windmagnetosphere-ionosphere system. Determining the dominant physical quantities controlling these currents is therefore important for understanding the transport of electromagnetic energy and momentum. The importance of the solar wind density and speed (Iijima and Potemra, 1982) and the interplanetary magnetic field (IMF) orientation (Potemra et al., 1984; Zanetti et al., 1984) in governing the Birkeland currents has been known for decades. Subsequent, comprehensive observations from various single satellite studies combined with upstream solar wind and IMF measurements provided quantitative, two-dimensional distributions of the large-scale Birkeland currents. Statistical studies of magnetic field observations by the Dynamics Explorer 2 (Weimer, 2001, 2005) and the Ørsted (Papitashvili et al., 2002) satellites detailed the IMF dependence of the current distributions, revealing the IMF direction as the fundamental quantity determining the global-scale coupling geometry.

With the advent of distributed observations of the largescale Birkeland currents by the constellation of Iridium satellites (Anderson et al., 2000), the capabilities for specifying the state of the system and relating this to solar wind conditions have significantly increased. The Iridium constellation consists of over 66 satellites in six 780-km altitude, circular polar orbit planes. Each satellite is equipped with an

Published by Copernicus Publications on behalf of the European Geosciences Union. 
engineering magnetometer digitized to $30-\mathrm{nT}$ resolution. For the period of this study these data were reported to the ground one vector magnetic field sample every $200 \mathrm{~s}$ on average. The temporal coverage of these observations is nearly continuous since February 1999. The Birkeland current distributions are calculated using Ampère's law from fits of spherical harmonic basis functions to the observed magnetic perturbations (Waters et al., 2001). To resolve Birkeland currents to $4^{\circ}$ in latitude, a data accumulation over about one hour is required (Korth et al., 2004b, 2008). The Iridium data used here span $\sim 75000 \mathrm{~h}$ of observations, allowing unprecedented statistical analyses of the Birkeland currents and their dependence on solar wind parameters.

The Iridium database provides unique advantages for the statistical characterization of the Birkeland currents. Recently, Anderson et al. (2008), hereinafter denoted P1, compiled statistical current distributions organized by IMF clock angle orientation. The statistical distributions were compiled from intervals with "stable" current topology obtained between February 1999 and December 2005. The selection of data by stability in the analysis distinguishes this work from previous statistical studies (Weimer, 2001, 2005; Papitashvili et al., 2002), which required using data from all data intervals without regard to stability to obtain global observational coverage. The topology of the Birkeland currents computed by averaging individual distributions derived from Iridium observations and sorted by IMF orientation agrees favorably with previous statistical analyses, but shows important differences. For example, the influence of the IMF $B_{\mathrm{y}}$, which governs the direction of high-latitude flows arising from magnetopause reconnection, on the configuration of the dayside Birkeland currents is consistent with previous studies conducted in the Northern Hemisphere. The effect of the sign of IMF $B_{\mathrm{y}}$ on the Birkeland current topology is reversed in the Southern Hemisphere due to the anti-symmetry of the reconnection site with respect to the noon-midnight meridian (Green et al., 2009). For southward IMF, the Birkeland current distributions derived from Iridium data do not show significant currents poleward of $80^{\circ}$ MLAT, whereas some previous results show statistically significant NBZ-sense currents (Zanetti et al., 1984) for all IMF orientations. Furthermore, for northward IMF, the Region-1 and particularly the Region- 2 currents are much smaller in area and intensity compared to previous models. P1 suggested that the luxury of restricting the Iridium analysis to those intervals with relatively stable currents yields distributions that more nearly reflect pure states of the magnetosphere-ionosphere system.

The results of P1 demonstrate the suitability of the Iridium stable-currents database for assessing the role of the IMF in governing solar wind-magnetosphere-ionosphere coupling. In this paper, this database is used to determine the influence of the intensity on the solar wind electric field, dynamic pressure, and Alfvén Mach number on the current distributions and intensities. Ultimately these quantitative relationships can be applied to evaluate global geospace simulations, in which the field-aligned current density is a fundamental quantity, to determine what aspects of Birkeland current dynamics are best and least understood. The analysis described here is a step toward the ultimate goal of constructing a comprehensive model of the solar wind-magnetosphereionosphere interaction. The statistical analyses are described in Sect. 2. The results are discussed in Sect. 3 and summarized in Sect. 4.

\section{Data analysis}

\subsection{Iridium database}

This study uses Iridium data from February 1999 to December 2007, two more years than used in P1. Iridium data were obtained for $97 \%$ of the days in this interval, yielding $\sim 75000$ two-dimensional current patterns for analysis. The Iridium magnetometer samples were first reduced to magnetic perturbation data as described by Anderson et al. (2000). The Birkeland currents were then derived by fitting these perturbation data using a set of spherical harmonic basis functions and applying Ampère's law to the fit (Waters et al., 2001). The spherical harmonic fits were computed in a custom spherical coordinate system with the intersection of the Iridium orbit planes at the pole. The results were transformed into Altitude Adjusted Corrected Geomagnetic (AACGM) coordinates (Baker and Wing, 1989), which are used throughout this manuscript. The maximum degree and order of the basis functions, and thus the spatial resolution of the current density, are prescribed by the spatial distribution of the data samples. In azimuth, the smallest wavelength must be at least twice the longitude spacing between the orbit planes, $\sim 30^{\circ}$. The latitude resolution afforded by the fit is a function of the magnetometer data sampling interval and the time over which data are accumulated. A sampling rate of one sample per $200 \mathrm{~s}$ from an individual satellite corresponds to a spatial along-track separation of $\sim 12^{\circ}$. The phasing of the magnetic field samples between satellites is random, and there are eleven satellites in each orbit plane, equally spaced along track, so the data point density along the orbit increases with time as samples from different satellites are made. For a one-hour data accumulation, the average spacing of the samples is less than $12^{\circ} \cdot 9 \mathrm{~min} / 60 \mathrm{~min}=1.8^{\circ}$. This allows the Birkeland current distributions to be recovered with a latitude resolution of $4^{\circ}$.

The analysis presented here includes only Northern Hemisphere Birkeland currents. The spherical harmonic fit of the cross-track magnetic field component exhibits a singularity at the orbit intersection point. Because of the eccentricity of the Earth's magnetic field, the orbit crossing point is frequently located within the Southern Hemisphere large-scale currents leading to less reliable current densities (P1). While improved estimates for the Southern Hemisphere Birkeland currents can be obtained (Green et al., 2008) using the 
Elementary Current Method (ECM) (Amm, 2001), following P1, we restricted our analysis to the Northern Hemisphere Birkeland current observations.

Solar wind plasma (McComas et al., 1998) and IMF (Smith et al., 1998) observations by the Advanced Composition Explorer (ACE) satellite at the first Lagrangian point, L1, were used to specify the solar wind/IMF conditions. For each one-hour Birkeland current distribution we averaged the ACE solar wind and magnetic field data for the time interval corresponding to simple advection delay between ACE and Earth. Since the intervals used are both long relative to errors in the advection time and correspond to stable IMF and solar wind properties (P1), this estimation for IMF conditions imposed on the system should be reliable.

\subsection{Event selection}

The magnetosphere can exhibit large-scale dynamics on time scales that are shorter than the one-hour accumulation times required to obtain Birkeland currents from the Iridium data used here. Care must therefore be taken in the selection of time intervals to be included in the analysis. Intervals were selected from the database for the statistical analysis using the method described in P1 to identify "stable" current distributions. Briefly, this technique uses image object identification to locate the dominant upward and downward current regions in two consecutive one-hour distributions and then determines the fractional overlap of both the upward and the downward current regions between the two distributions. Requiring a fractional overlap of at least $45 \%$ ensured that the automatic procedure selected only those pairs of distributions that one would visually identify as consistent. The resulting stable-current intervals also correspond to stable conditions in the solar wind (P1). A total of 1536 events, corresponding to $4 \%$ of the database, met the selection criterion and have simultaneous observations of both IMF and solar wind plasma from ACE. Since plasma observations are available for a slightly smaller fraction of the time than IMF data, the fraction of suitable events available here is somewhat lower than in P1.

\subsection{Conductance normalization}

Systematic variations in ionospheric conductivity also change the Birkeland currents and the solar wind control of the currents must be distinguished from conductance effects. The two main sources of ionization are solar extreme ultraviolet (EUV) radiation and energetic particle precipitation. The distributions of particle precipitation cannot be measured directly and must either be inferred from auroral luminosities (Germany et al., 1989) or taken from statistical models derived from auroral (e.g., Zhang and Paxton, 2008) or precipitation observations (e.g., Hardy et al., 1987, 1989). However, the uncertainties associated with each of these methods are significant so that one risks introducing greater system- atic errors by applying a correction than one might hope to remove. Moreover, the discrete precipitation is closely associated with Birkeland currents, so that the distribution of currents and precipitation generally vary coherently. Consequently, introducing a statistical correction for particle precipitation that does not move with the currents is likely to be worse than doing nothing. In the present analysis, we therefore account only for changes in conductance due to solar illumination.

The Birkeland currents are affected primarily by the Pedersen conductance, $\Sigma_{\mathrm{P}}$, and Green et al. (2009) have shown that the current densities on the dayside are markedly reduced during the winter months when the conductance due to solar EUV is generally lower. The solar EUV influence on $\Sigma_{\mathrm{P}}$ can be expressed in terms of the solar zenith angle as (Rasmussen et al., 1988):

$\Sigma_{\mathrm{P}}=4.5\left(1-0.85 v^{2}\right)\left(1+0.15 u+0.05 u^{2}\right) / B$,

where $v=\chi / 90, u=F_{10.7} / 90, B$ is the magnetic field strength, $F_{10.7}$ is the $10.7-\mathrm{cm}$ solar radio flux, and $\chi$ is the solar zenith angle. We normalized the dayside current densities to equinox conditions by multiplying each Birkeland current distribution, $j_{i}(\theta, \phi)$, where the index $i$ denotes the event number assigned to the current distribution, by the ratio between the conductance distributions at equinox, $\Sigma_{\mathrm{P} \text {,eq }}(\theta, \phi)$, and at the time of the observations, $\Sigma_{\mathrm{P}, i}(\theta, \phi)$. The normalized current density, $j_{i}^{*}(\theta, \phi)$ is given by:

$j_{i}^{*}(\theta, \phi)=j_{i}(\theta, \phi) \cdot \Sigma_{\mathrm{P}, \mathrm{eq}}(\theta, \phi) / \Sigma_{\mathrm{P}, \mathrm{i}}(\theta, \phi)$,

where $\theta$ and $\phi$ are the magnetic colatitude and local time, respectively. For the equinox conductance distribution, we assume a solar radio flux of $F_{10.7}=150 \times 10^{-22} \mathrm{Wm}^{2} \mathrm{~Hz}^{-1}$.

Equation (1) is applicable within the solar zenith angle range $0^{\circ} \leq \chi \leq 85^{\circ}$. For $\chi>85^{\circ}$, that is near the terminator and on the nightside, we do not anticipate a variation in conductance due to EUV so the normalization ratio should be set to unity there. To do this in a continuous way we first evaluated the minimum in $\Sigma_{\mathrm{P}, \text { eq }}$ and in $\Sigma_{\mathrm{P}}$ at $\chi=85^{\circ}$. We then used the larger of these two minima as $\Sigma_{\mathrm{P} \text {,min }}$ and set all values in $\Sigma_{\mathrm{P} \text {,eq }}$ and $\Sigma_{\mathrm{P}}$ that were below $\Sigma_{\mathrm{P} \text {,min }}$ equal to $\Sigma_{\mathrm{P}, \min }$. The ratio $\Sigma_{\mathrm{P}, \mathrm{eq}} / \Sigma_{\mathrm{P}, \mathrm{i}}$ then transitions smoothly to unity at the terminator and the current densities on the nightside are unaffected by the normalization.

\subsection{Solar wind electric field dependence}

The spatial distributions of the currents are governed primarily by the IMF clock angle, $\alpha=\arctan \left(B_{\mathrm{y}} / B_{\mathrm{z}}\right)(\mathrm{P} 1)$ so we first divided the data set by $\alpha$ into $45^{\circ}$-wide bins of the IMF clock angle centered at $\alpha=0^{\circ}, 45^{\circ}, 90^{\circ}, 135^{\circ}, 180^{\circ}, 225^{\circ}$, $270^{\circ}$, and $315^{\circ}$. Within each clock angle bin we analyzed the Birkeland current distributions normalized to equinox illumination conditions for any dependence on the magnitude 
of the solar wind electric field, $E_{\mathrm{yz}}$. For each of the distributions we computed $E_{\mathrm{yz}}=v_{\mathrm{p}} \sqrt{B_{\mathrm{y}}^{2}+B_{\mathrm{z}}^{2}}$, where $v_{\mathrm{p}}$ is the proton speed and $B_{\mathrm{y}}$ and $B_{\mathrm{z}}$ are components of the IMF in GSM coordinates. The events within each $\alpha$ bin were separated by the magnitude of $E_{\mathrm{yz}}$ into $1 \mathrm{mV} \mathrm{m}^{-1}$-wide bins centered at $E_{\mathrm{yz}}=1,2,3$, and $4 \mathrm{mV} \mathrm{m}^{-1}$. For $E_{\mathrm{yz}}>4.5 \mathrm{mV} \mathrm{m}^{-1}$, the number of events was insufficient for statistical analysis, so we restricted the study to $E_{\mathrm{yz}}<=4.5 \mathrm{mV} \mathrm{m}^{-1}$, corresponding to low and moderate solar wind electric fields.

The average distributions within each $\left(E_{\mathrm{yz}}, \alpha\right)$ bin are shown in Fig. 1. The rows and columns represent bins of the IMF clock angle and solar wind electric field magnitude, respectively. The average magnitude of the total current (top) and the number of events within each bin (bottom) are listed to the right of each distribution. The Birkeland total current was calculated as

$I_{\mathrm{tot}}=\sum_{\theta=1}^{49} \sum_{\phi=0}^{23} \frac{|j(\theta, \phi)| \delta A}{2}$

where $\delta A=R_{\mathrm{i}}^{2} \delta \phi \delta \theta \sin \theta$ is the area element computed using $R_{\mathrm{i}}=6481 \mathrm{~km}$ for the radius of the ionosphere at an assumed altitude of $110 \mathrm{~km}, \delta \phi=1 \mathrm{~h}$, and $\delta \theta=2^{\circ}$. It is evident from Fig. 1 that the Birkeland current densities intensify for increasing $E_{\mathrm{yz}}$ and this is reflected in the total current. This trend is stronger for southward than northward IMF. In addition to the overall intensification of the Birkeland currents, the large-scale current regions obtained for southward IMF expand equatorward as $E_{\mathrm{yz}}$ increases.

Figure 2 shows scatter plots of $I_{\text {tot }}$ versus $E_{\mathrm{yz}}$ for each current pattern sorted by IMF clock angle. The statistical dependence of the Birkeland total current on $E_{\mathrm{yz}}$ within each $\alpha$-bin was estimated using a linear fit between $I_{\text {tot }}$ and $E_{\mathrm{yz}}$. The electric fields considered here are less than values where saturation effects occur (Siscoe et al., 2002b; Ober et al., 2003; Anderson and Korth, 2007). The linear fits are shown in Fig. 2 including the corresponding fit parameters and uncertainties. The Birkeland total current increases with $E_{\mathrm{yz}}$ for all IMF clock angles. The linear fit slope depends on IMF orientation and is smallest for northward IMF, where the slope magnitude is less than the $1-\sigma$ confidence threshold $\left(0.1 \mathrm{MA} /\left(\mathrm{mV} \mathrm{m}^{-1}\right)\right)$. The largest slope occurs for southward IMF $\left(0.8 \mathrm{MA} /\left(\mathrm{mV} \mathrm{m}^{-1}\right)\right)$. The dependence of the Birkeland total current on $E_{\mathrm{yz}}$ for all IMF clock angle bins is summarized in the second column of Table 1.

The distributions in Fig. 1 also change with $E_{\mathrm{yz}}$. Figure 3 shows the current density along the dawn-dusk meridian for the four $E_{\mathrm{yz}}$ levels in the $180^{\circ}$ clock angle bin. An equatorward expansion of the currents with increasing $E_{\mathrm{yz}}$ is clearly evident.

To quantify the influence of $E_{\mathrm{yz}}$ on the patterns, the average distributions within each IMF clock angle bin for different $E_{\mathrm{yz}}$ magnitudes were compared to the $E_{\mathrm{yz}}=2 \mathrm{mV} \mathrm{m}^{-1}$ distribution for that clock angle bin. For this analysis we
Table 1. Dependence of the Birkeland total current, $I_{\text {tot }}$, on $E_{\mathrm{yz}}$, $p_{\mathrm{sw}}$, and $M_{\mathrm{A}}$ for different IMF clock angles. All uncertainties quoted are those of the 1- $\sigma$ confidence level.

\begin{tabular}{cccc}
\hline $\begin{array}{c}\text { Clock angle } \\
{\left[{ }^{\circ}\right]}\end{array}$ & $\begin{array}{c}I_{\text {tot }} / E_{\mathrm{yz}} \\
{\left[\mathrm{MA} /\left(\mathrm{mVm}^{-1}\right)\right]}\end{array}$ & $\begin{array}{c}I_{\mathrm{tot}} / p_{\mathrm{sw}} \\
{[\mathrm{MA} / \mathrm{nPa}]}\end{array}$ & $\begin{array}{c}I_{\mathrm{tot}} / M_{\mathrm{A}} \\
{\left[\mathrm{MA} / \mathrm{unitM}_{\mathrm{A}}\right]}\end{array}$ \\
\hline 0 & $-0.08 \pm 0.10$ & $0.28 \pm 0.04$ & $0.01 \pm 0.02$ \\
45 & $0.05 \pm 0.04$ & $0.05 \pm 0.03$ & $-0.03 \pm 0.01$ \\
90 & $0.39 \pm 0.06$ & $0.19 \pm 0.03$ & $0.04 \pm 0.01$ \\
135 & $0.69 \pm 0.06$ & $0.22 \pm 0.05$ & $0.03 \pm 0.02$ \\
180 & $0.79 \pm 0.06$ & $0.44 \pm 0.06$ & $0.05 \pm 0.02$ \\
225 & $0.68 \pm 0.07$ & $0.14 \pm 0.05$ & $0.04 \pm 0.02$ \\
270 & $0.17 \pm 0.08$ & $0.12 \pm 0.05$ & $0.04 \pm 0.02$ \\
315 & $0.22 \pm 0.05$ & $0.25 \pm 0.03$ & $0.00 \pm 0.01$ \\
\hline
\end{tabular}

used the distribution of the average horizontal magnetic perturbation, $\delta B$, rather than the current density. The smoother structure of the magnetic perturbations provides more stable root mean differences between patterns, and hence less noise in the differences between patterns, so that the shifts in the patterns can be computed more reliably.

In principle it is possible that changes in the location of the $\delta B$ distribution can occur if only some of the currents change their locations. This might be the case for example if only the Region-2 currents shift equatorward with increasing $E_{\mathrm{yz}}$. If the latitude spanned by the current system does not change self-similarly with the equatorward displacement of the currents, this will show up as a systematic distribution in the $\delta B$ residuals relative to the reference distribution. The $\delta B$ residuals would then exhibit two rings, one poleward of the reference Region- 1 currents, and the second equatorward of the reference Region 2 currents. Thus, by examining the 2-D magnetic perturbation residuals for systematic patterns we check for indications of such behavior. We note that the dawn-dusk meridian cuts in Fig. 3 do not show evidence of such behavior and none is found in the 2-D distributions either.

For this analysis the distribution for $E_{\mathrm{yz}}=2 \mathrm{mV} \mathrm{m}^{-1}$ was chosen as the reference $\delta B$ pattern and the grid positions of the magnetic perturbations for other $E_{\mathrm{yz}}$ distributions were parameterized with a colatitude expansion factor and offsets in the midnight-to-noon and dawn-to-dusk direction. The best-fit latitudinal expansion factor and noonward and dawnward shift were determined simultaneously by minimizing the root-mean-square (rms) of the vector magnetic field residuals relative to the reference distribution.

Figure 4 shows the colatitude expansion as a function of $E_{\mathrm{yz}}$ for all IMF clock angle bins with $B_{\mathrm{z}} \leq 0 \mathrm{nT}$, and the expansion factors are summarized in the second column of Table 2. The currents expand equatorward with increasing $E_{\mathrm{yz}}$. The colatitude expansion per unit electric field is largest for southward IMF and decreases as the IMF $B_{\mathrm{y}}$ becomes more dominant. The average equatorward expansion of the 


\section{Solar Wind Electric Field Eyz [mV/m]}

1.0

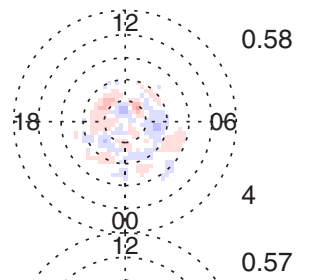

45

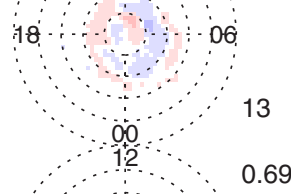

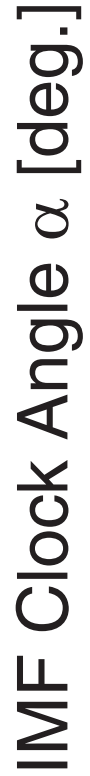

90

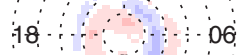

(1) 135
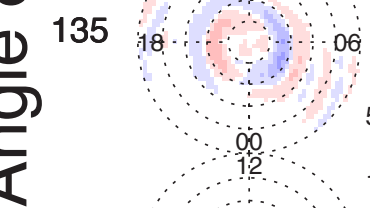

180
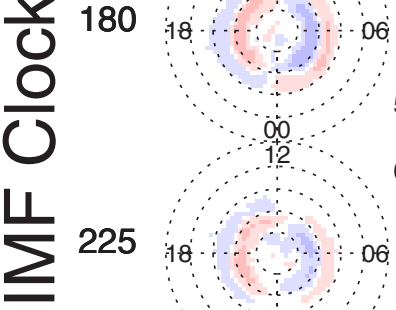

225

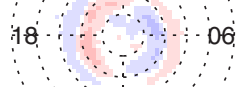

50

0

1.19

270

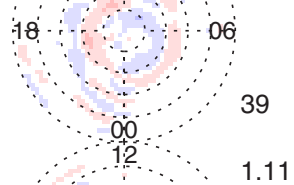

315

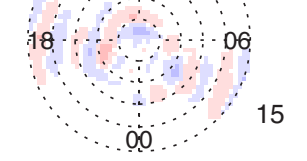

0.96

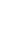

2.0

$\cdots 12 \cdots, 0.60$

1800

$\begin{array}{lll}00 & 12 \\ 12 & 0 & \\ 12 & & \end{array}$

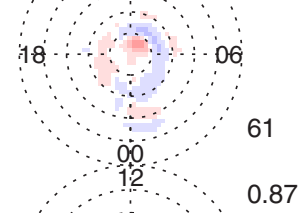

0.87

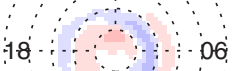

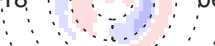

00116

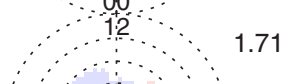

$18-10$

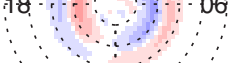

00116

00

1.85

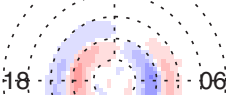

18
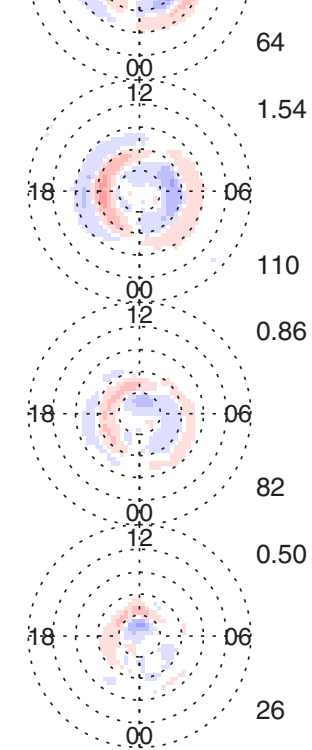

Birkeland Current Density $\left[\mu \mathrm{A} / \mathrm{m}^{2}\right]$

\begin{tabular}{lllll}
\hline & & & & \\
\hline-1.00 & -0.50 & 0.00 & 0.50 & 1.00
\end{tabular}
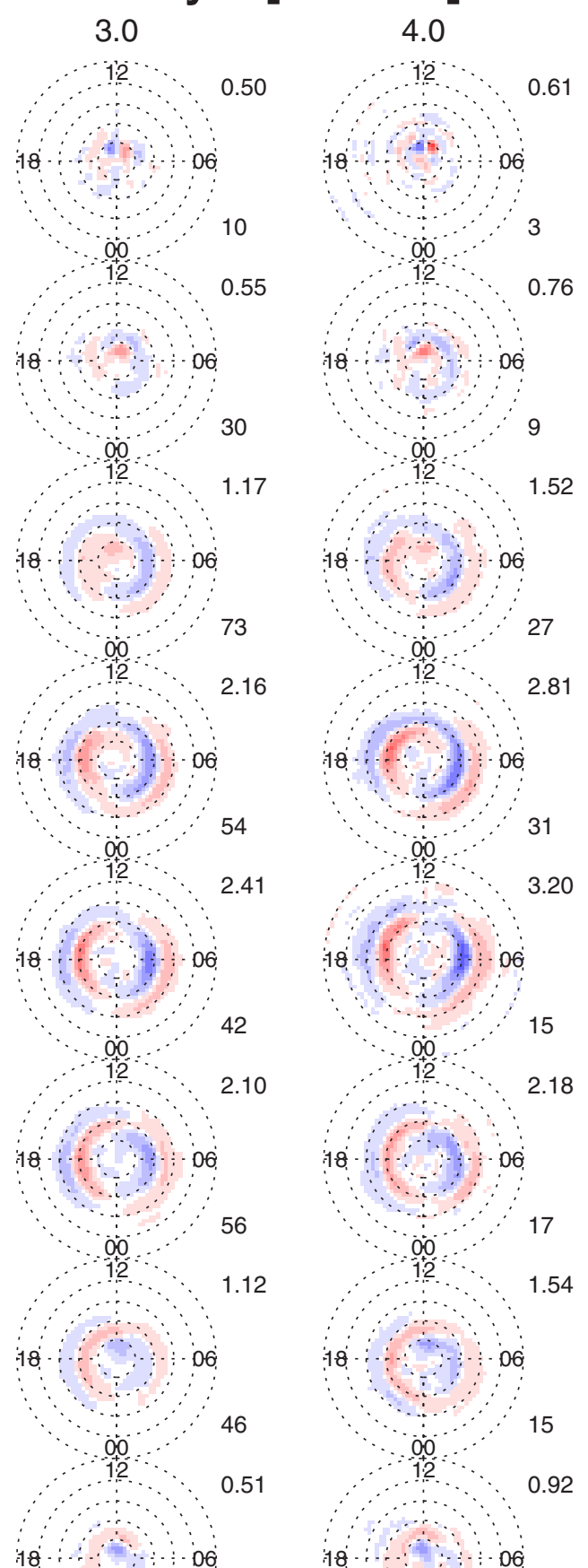

34
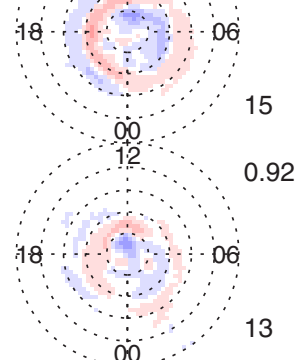

OO०

\section{8}



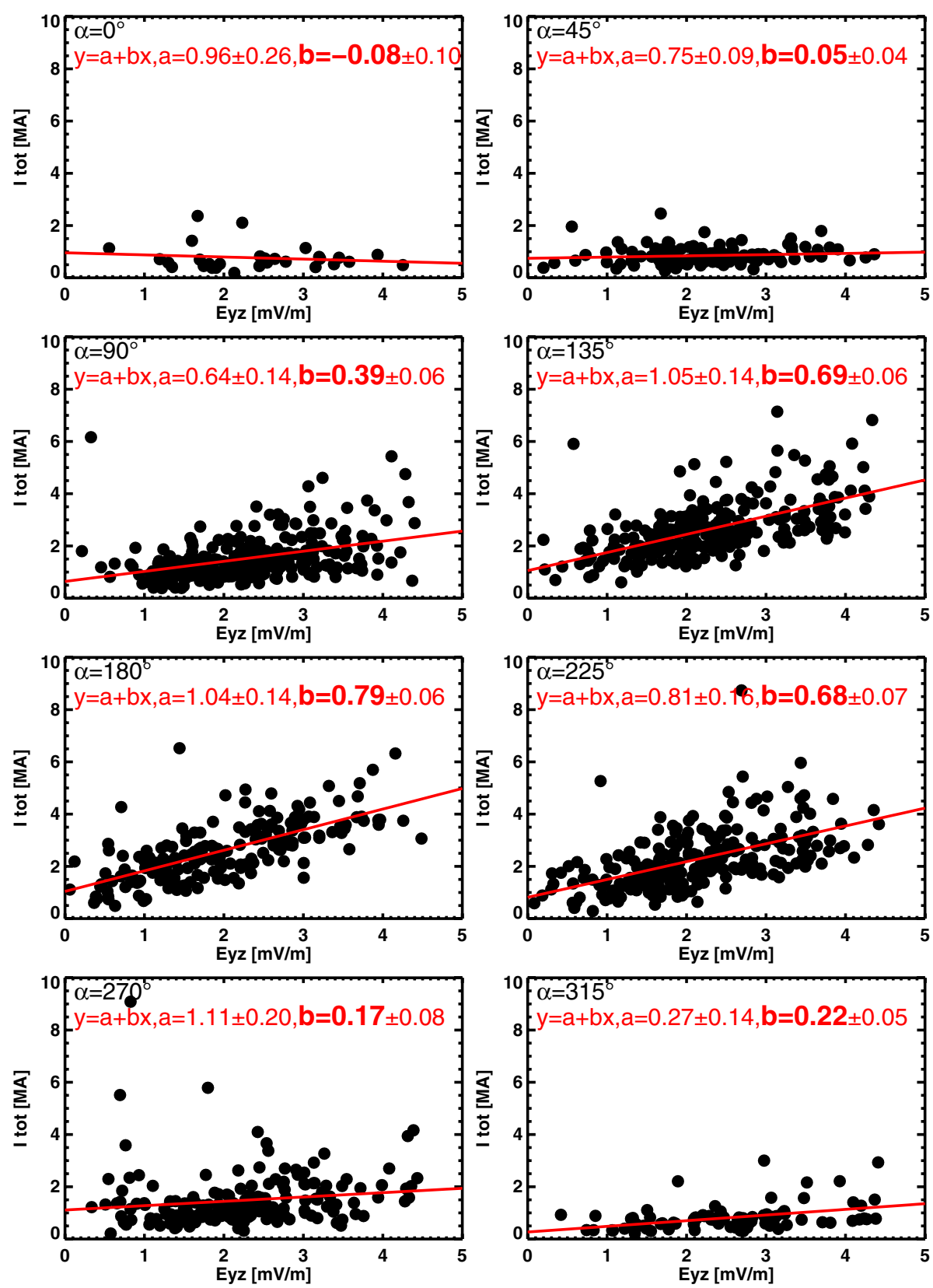

Fig. 2. Dependence of the Birkeland total current on the solar wind electric field, $E_{\mathrm{yz}}$, and IMF clock angle, $\alpha$. The parameters and 1- $\sigma$ uncertainties of the linear fits (red lines) of these data are given at the top of each panel.

Birkeland currents for the IMF clock angle range $90^{\circ} \leq \alpha \leq$ $270^{\circ}$ is $3 \% /\left(\mathrm{mV} \mathrm{m}^{-1}\right)$. The shifts of the large-scale currents along the noon-midnight and dawn-dusk meridians are presented in Fig. 5. Since the translation of the current patterns did not show a dependence on IMF clock angle a single linear function fits were estimated for the noonward and dawnward direction from all shifts evaluated in the clock angle range $90^{\circ} \leq \alpha \leq 270^{\circ}$ at a particular $E_{\mathrm{yz}}$ level. We find that the currents shift toward local noon with increas- ing $E_{\mathrm{yz}}$ by about $0.4^{\circ} /\left(\mathrm{mVm}^{-1}\right)$, with an uncertainty in the fitted slope of $0.1^{\circ} /\left(\mathrm{mVm}^{-1}\right)$ (cf. Fig. 5, top panel). On the other hand, the slope of fit for the dawnward shift, shown in the bottom panel of Fig. 5, is below the 1- $\sigma$ confidence level. A shift in the dawn-dusk direction was thus not detected. Therefore, given the relative colatitude expansion function $s=1+0.03 \delta E_{\mathrm{yz}}$ and noon shift function $c=0.4 \delta E_{\mathrm{yz}}$, where $\delta$ denotes the change in $E_{\mathrm{yz}}$, the $E_{\mathrm{yz}^{-}}$ dependent transformation of the Birkeland current colatitude 


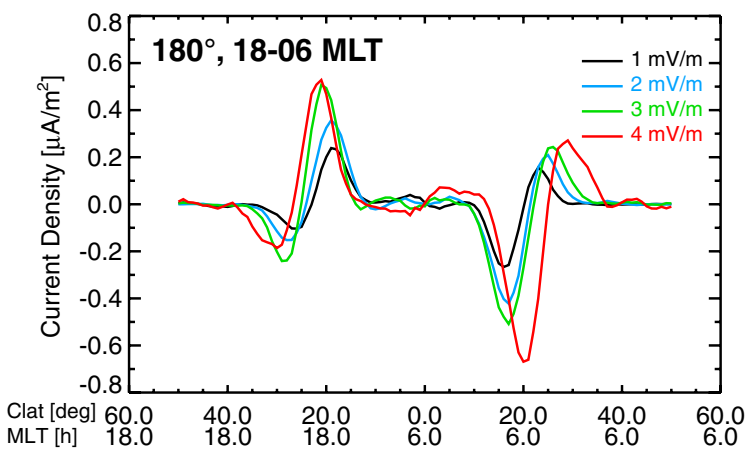

Fig. 3. Birkeland current density along the dawn-dusk (18:0006:00 MLT) meridian for $E_{\mathrm{yz}}=1$ (black), 2 (blue), 3 (green), and $4 \mathrm{mV} \mathrm{m}^{-1}$ (red) in the $180^{\circ}$ clock angle bin.

Table 2. Colatitude expansion factors of the Birkeland currents for $E_{\mathrm{yz}}, p_{\mathrm{sw}}$, and $M_{\mathrm{A}}$ sorted by IMF clock angle. All uncertainties quoted are those of the $1-\sigma$ confidence level.

\begin{tabular}{cccc}
\hline $\begin{array}{c}\text { Clock angle } \\
{\left[{ }^{\circ}\right]}\end{array}$ & $\begin{array}{c}E_{\mathrm{yz}} \\
{\left[\% /\left(\mathrm{mVm}^{-1}\right)\right]}\end{array}$ & $\begin{array}{c}p_{\mathrm{sw}} \\
{[\% / \mathrm{nPa}]}\end{array}$ & $\begin{array}{c}M_{\mathrm{A}} \\
{\left[\% / \mathrm{unitM}_{\mathrm{A}}\right]}\end{array}$ \\
\hline 90 & $2.7 \pm 0.3$ & $3.7 \pm 0.5$ & $1.3 \pm 0.2$ \\
135 & $2.5 \pm 0.4$ & $1.2 \pm 1.1$ & $0.1 \pm 0.5$ \\
180 & $5.5 \pm 1.1$ & $0.7 \pm 0.2$ & $-0.4 \pm 0.3$ \\
225 & $2.5 \pm 0.5$ & $1.5 \pm 0.6$ & $0.9 \pm 0.1$ \\
270 & $1.4 \pm 0.4$ & $1.6 \pm 0.4$ & $0.7 \pm 0.1$ \\
\hline
\end{tabular}

and local time, $\theta$ and $\phi$, is given by:

$\theta^{*}=\sqrt{s^{2} \theta^{2}+2 s \theta \sin [15(\phi-6)]+c^{2}}$,

$\phi^{*}=\arctan \left(\frac{s \theta \sin [15(\phi-6)]+c}{s \theta \cos [15(\phi-6)]}\right)$.

The shift of the large-scale currents toward noon is not immediately evident from Fig. 1. Figure 6 shows the Birkeland current density along the 03:00-15:00 MLT (top panel) and 21:00-09:00 MLT (bottom panel) meridians for each $E_{\mathrm{yz}}$ level in the $180^{\circ}$ clock angle bin. The distributions used to evaluate these cuts were corrected for the equatorward expansion of the Birkeland currents using the scale factor derived above. The latitudes of the nightside currents do not depend $E_{\mathrm{yz}}$ but the dayside currents move equatorward with increasing $E_{\mathrm{yz}}$, demonstrating the shift toward noon identified above.

For IMF $B_{\mathrm{Z}}>0$, colatitude expansion and noon shift cannot be determined reliably for two reasons. First, fewer events are available in each $\left(E_{\mathrm{yz}}, \alpha\right)$ bin for these conditions, and the resulting statistical distributions are correspondingly less well defined so that the variance between distributions is dominated by the scatter and systematic trends are not apparent. Second, the Birkeland currents are concentrated at high latitudes and their areas are significantly smaller than for

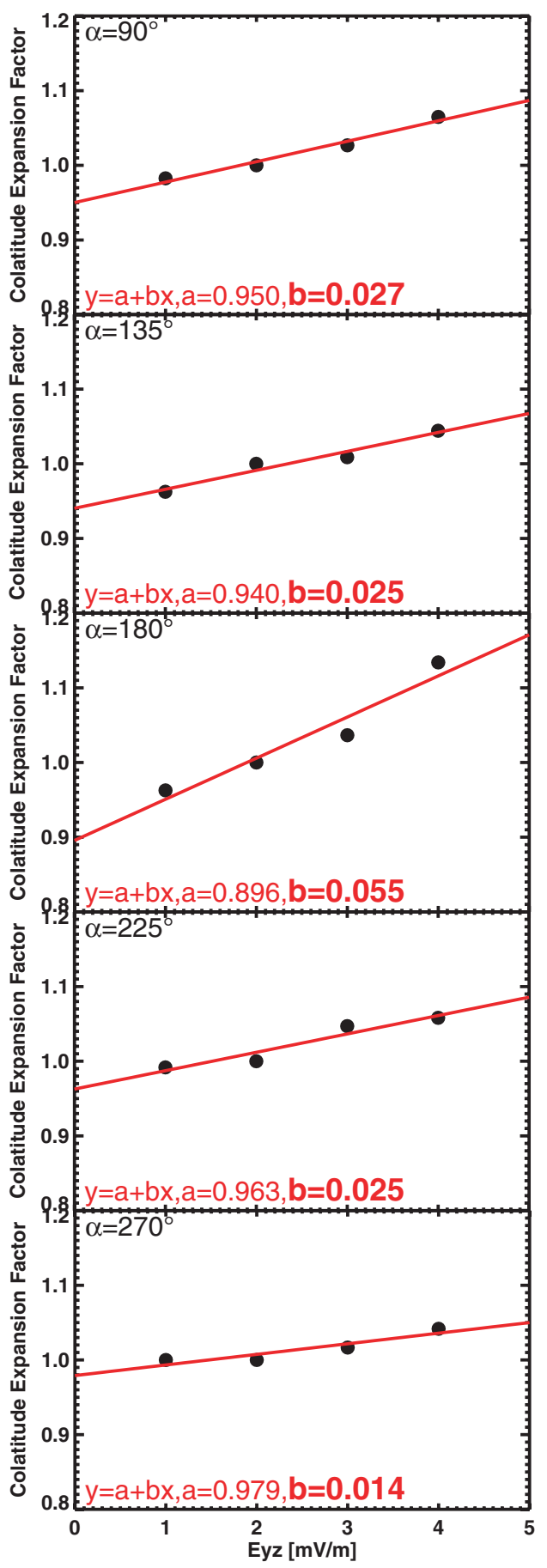

Fig. 4. Dependence of the colatitude expansion factor on the solar wind electric field, $E_{\mathrm{yz}}$, and IMF clock angle, $\alpha$. The parameters and $1-\sigma$ uncertainties of the linear fits (red lines) of these data are given at the bottom of each panel.

southward IMF. Hence, the corresponding magnetic perturbations are represented by fewer grid points in the database. In the comparison between patterns used to estimate the 


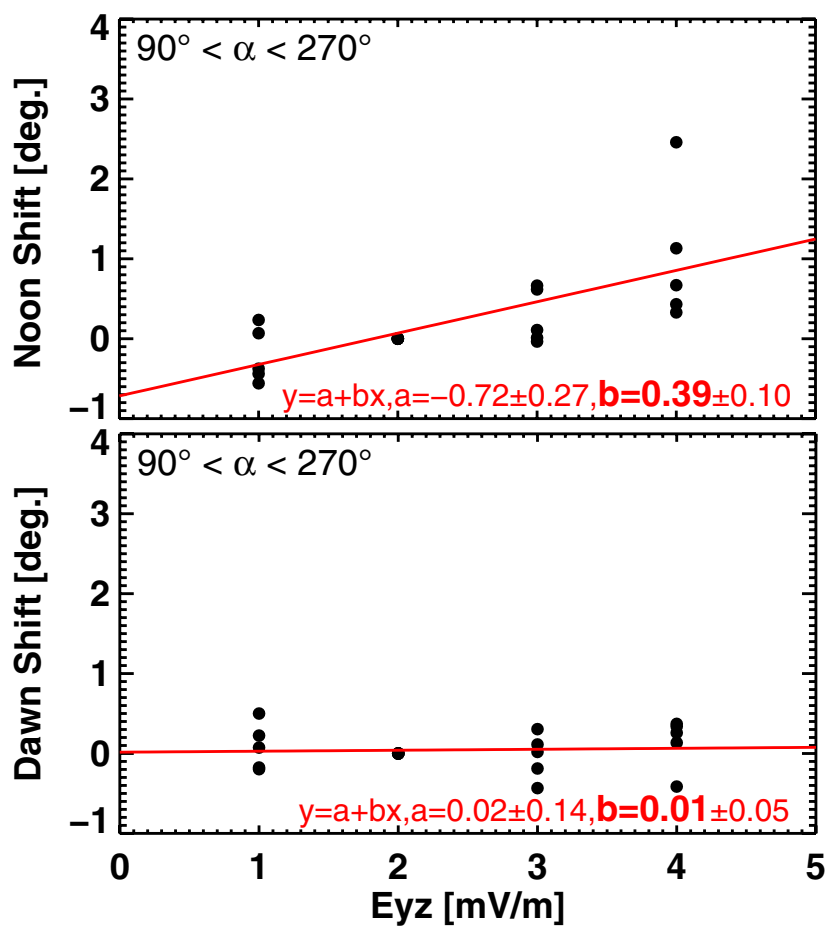

Fig. 5. Noon-midnight (top panel) and dawn-dusk (bottom panel) translation as function of the solar wind electric field. The parameters and 1- $\sigma$ uncertainties of the linear fits (red lines) of these data are given at the bottom of each panel.

relative spatial distributions, it turns out that the noise in regions without currents dominates the deviation so that the optimization algorithm fails to yield reliable results.

\subsection{Solar wind dynamic pressure dependence}

The solar wind pressure, for constant $E_{\mathrm{yz}}$, affects the size of the magnetosphere (Sotirelis and Meng, 1999) and the magnetospheric plasma densities (Wing and Newell, 1998; Borovsky et al., 1998), both of which might be expected to influence the Birkeland currents. To analyze the influence of the solar wind dynamic pressure, $p_{\mathrm{sw}}$, we first removed the dependence on $E_{\mathrm{yz}}$ by normalizing the Birkeland current distributions to a mean $E_{\mathrm{yz}}$ of $2.3 \mathrm{mV} \mathrm{m}^{-1}$ using the relationships given in Sect. 2.4. The current patterns were separated into the IMF clock angle bins used above and then sorted by solar wind dynamic pressure within each IMF clock angle bin. The solar wind dynamic pressure was calculated as $p_{\text {sw }}=m_{\mathrm{p}} n_{\mathrm{p}} v_{\mathrm{p}}^{2}$, where $m_{\mathrm{p}}$ is the proton mass and $n_{\mathrm{p}}$ is the proton density measured by ACE. The data set was binned by solar wind dynamic pressure in four one $\mathrm{nPa}$-wide bins centered at $0.5,1.5,2.5$, and $3.5 \mathrm{nPa}$.

The average current patterns for each $\left(p_{\mathrm{sw}}, \alpha\right)$ bin are shown in Fig. 7. There is an overall increase in the current densities with increasing $p_{\mathrm{sw}}$. The dependence of Birkeland

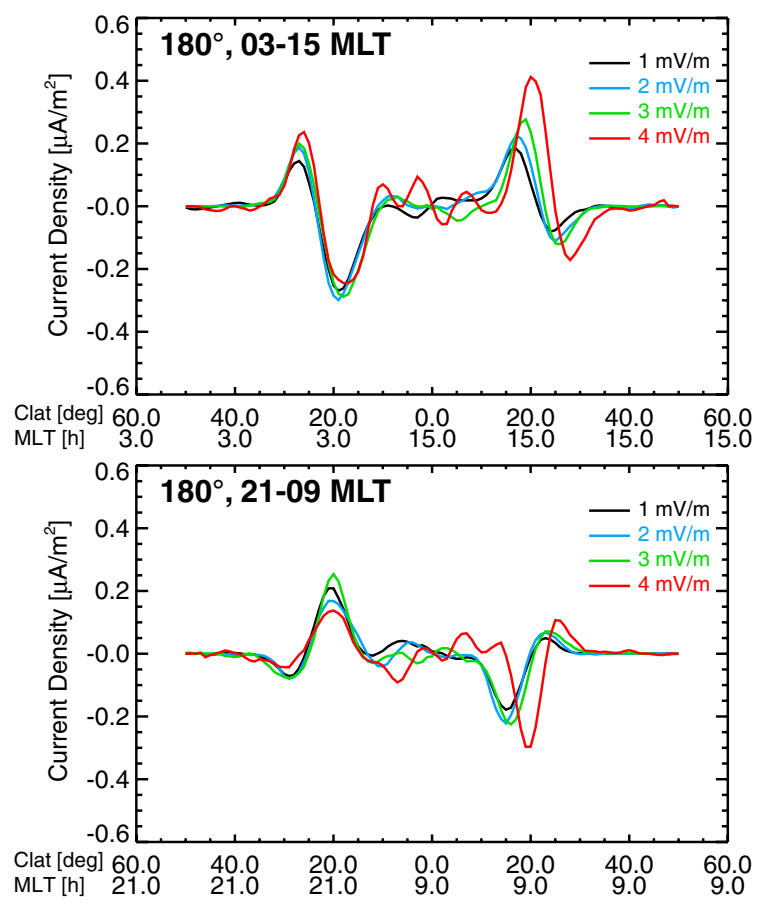

Fig. 6. Birkeland current density along the 03:00-15:00 MLT (top panel) and 21:00-09:00 MLT (bottom panel) meridians for $E_{\mathrm{yz}}=1$ (black), 2 (blue), 3 (green), and $4 \mathrm{mV} \mathrm{m}^{-1}$ (red) in the $180^{\circ}$ clock angle bin. The colatitude is corrected for the equatorward expansion of the Birkeland currents.

total current on $p_{\mathrm{sw}}$ is presented in Fig. 8, which shows the total current as a function of dynamic pressure in each IMF clock angle bin. The results are summarized in the third column of Table 1. The linear trend lines show that the total current intensifies with increasing $p_{\mathrm{sw}}$, independent of IMF orientation. The maximum increase of the Birkeland total current per unit $p_{\text {sw }}$ is about $0.4 \mathrm{MA} / \mathrm{nPa}$ for southward IMF while local minima are found for dawnward and duskward IMF orientation. The influence of $p_{\mathrm{sw}}$ on the Birkeland current pattern is less evident. The factors for the equatorward expansion are shown in the third column of Table 2. Compared with the effect of the $E_{\mathrm{yz}}$ within a given clock angle bin having a non-zero southward IMF component, the equatorward expansions due to increases in $p_{\mathrm{sw}}$ are not only markedly smaller but also approach the confidence thresholds of the slopes from the linear trend lines. Nevertheless, the slopes are consistently positive and exhibit magnitudes above the $1-\sigma$ threshold so that the small $p_{\mathrm{sw}}$-driven equatorward expansion may be genuine.

\subsection{Solar wind Alfvén Mach number dependence}

The reconnection efficiency may depend on the magnetosheath plasma beta, which in turn can be related to the solar wind Alfvén Mach number (Anderson et al., 1997). 


\section{Solar Wind Dynamic Pressure $\mathrm{p}_{\mathrm{sw}}[\mathrm{nPa}]$ \\ 0.5 \\ 12 \\ 0.75 \\ 1.5 \\ 12 \\ 0.48 \\ 2.5 \\ 12 \\ 0.47

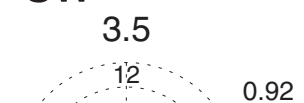

0

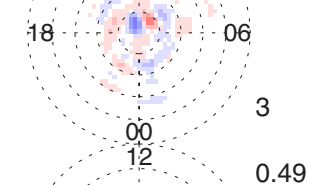

45

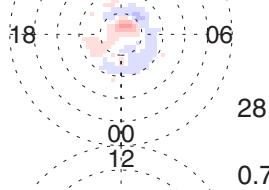

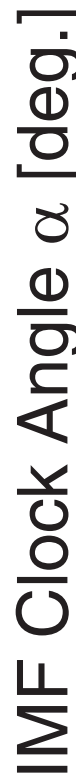

90

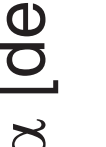

(1) 135

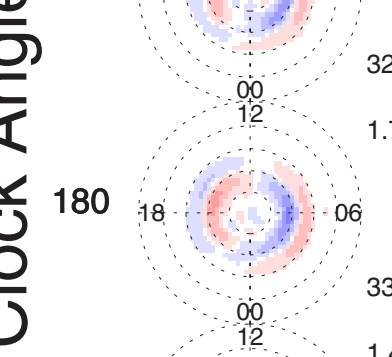

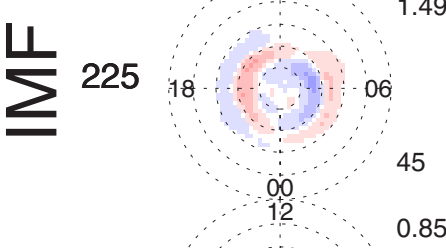

$\begin{array}{ll}00 & 51 \\ 12 & 1.68\end{array}$

1.68

32

1.77

06

.49
18000

10

0.76

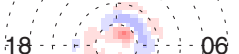

56

1.00

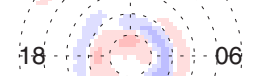

270

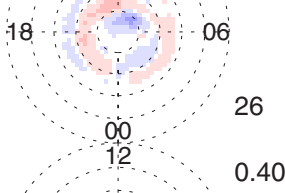

315

1.92

83

1.63

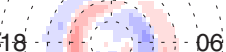

06

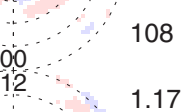

$18-106$

0.95

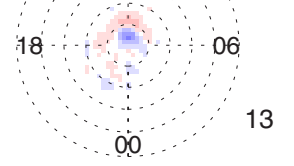

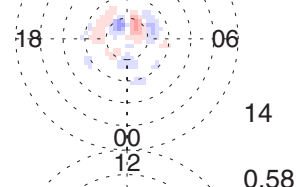

18

06

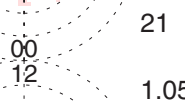

1.05

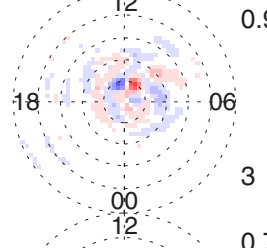

0.74

$18-106$ 0010

$1800 \quad 180$

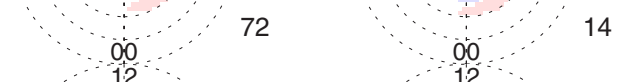

2.10
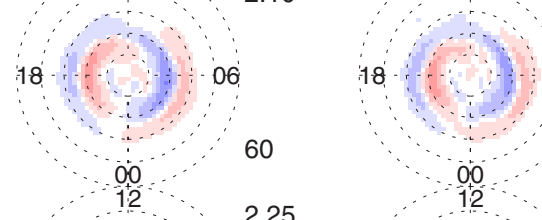

$-06$

31

00

2.61

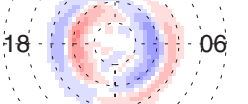

45

00

1.77
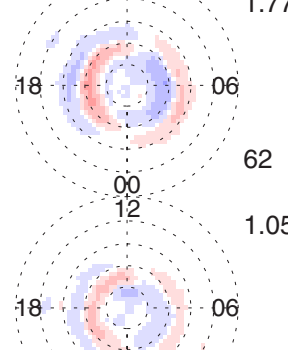

52
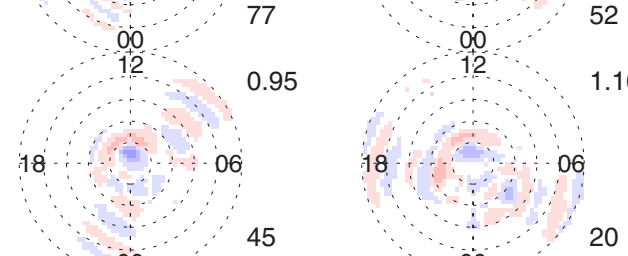

00

20

00

45

Birkeland Current Density $\left[\mu \mathrm{A} / \mathrm{m}^{2}\right]$

\begin{tabular}{|ccccc|}
\hline & & & & \\
\hline-1.00 & -0.50 & 0.00 & 0.50 & 1.00
\end{tabular}

1800

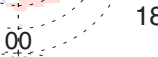

$12 \cdots 1.96$

96

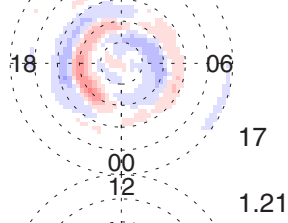

$18-06$

00

0.73

$18-06$

00

\section{8}

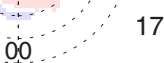



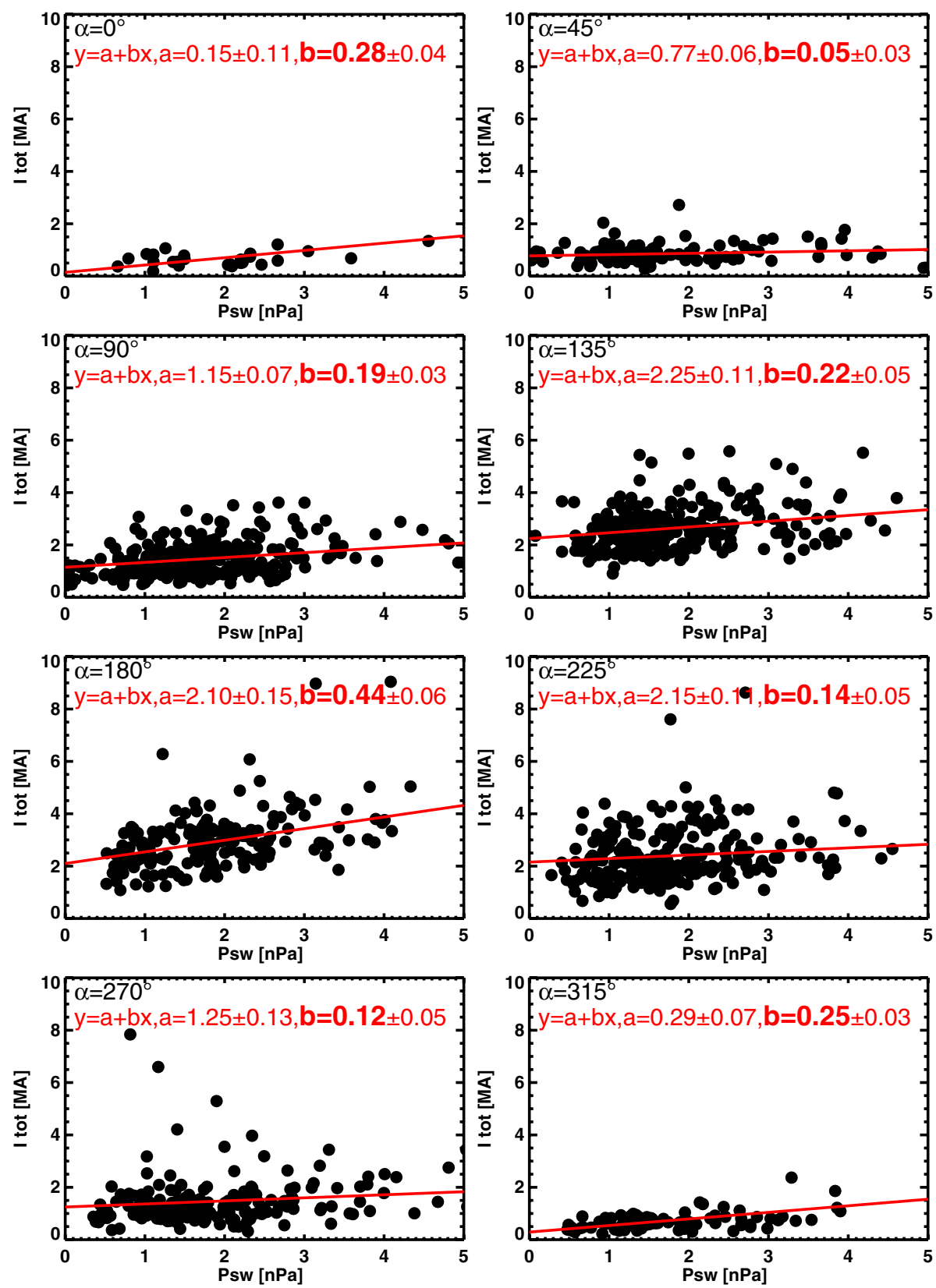

Fig. 8. Dependence of the Birkeland total current on the solar wind dynamic pressure, $p_{\mathrm{sw}}$, and IMF clock angle, $\alpha$. The parameters and $1-\sigma$ uncertainties of the linear fits (red lines) of these data are given at the top of each panel.

We therefore also examined the influence of the solar wind Alfvén Mach number on the Birkeland currents. The Birkeland currents were normalized to mean values of both solar wind electric field, $E_{\mathrm{yz}}=2.3 \mathrm{mV} \mathrm{m}^{-1}$, and dynamic pressure, $p_{\mathrm{sw}}=1.75 \mathrm{nPa}$, using the linear trends presented above. The solar wind Alfvén Mach number, $M_{\mathrm{A}}$, was calculated from ACE data as $M_{\mathrm{A}}=v_{\mathrm{p}} / v_{\mathrm{A}}$, where the Alfvén velocity $v_{\mathrm{A}}=B / \sqrt{\mu_{0} n_{\mathrm{p}} m_{\mathrm{p}}}$ was computed from the magnetic field intensity, $B$, and the proton mass, $m_{\mathrm{p}}$. The current patterns were sorted into $\left(M_{\mathrm{A}}, \alpha\right)$ bins 2.5 units wide and centered at $M_{\mathrm{A}}=2.5,5.0,7.5$, and 10.0. The resulting distributions (not shown) did not exhibit discernible variations in either the pattern or the current density on $M_{\mathrm{A}}$. The Birkeland total current is plotted versus $M_{\mathrm{A}}$ within each $\alpha$-bin in Fig. 9, and the linear fit slopes are summarized in the fourth column of Table 1. Linear fits to these data yield slopes that are mostly comparable to and in some cases lower in magnitude than the associated 1- $\sigma$ uncertainties. Considering that the total 
current shows consistent increases for all but one IMF clock angle bin, one could infer, if anything, a minor increase in $I_{\text {tot }}$ with increasing $M_{\mathrm{A}}$. In addition, the colatitude expansion factors associated with $M_{\mathrm{A}}$ (Table 2, fourth column), gave the smallest values in every clock angle bin indicating a very weak dependence. We conclude that the Iridium statistical distributions provide no evidence for significant control of the large-scale Birkeland currents by the solar wind Alfvén Mach number.

\subsection{Simultaneous multi-variable regression}

The parameters we chose to represent the solar wind influence on the magnetosphere were selected based on their physical roles in the magnetosphere-solar wind interaction. The clock angle is used because it plays a dominant role in controlling the location of magnetopause reconnection and hence on the configuration of the Birkeland currents. The solar wind electric field is the primary factor governing the intensity of reconnection and hence convection for a given clock angle. The solar wind ram pressure is the dominant factor determining the size of the magnetosphere, and finally the Alfvén Mach number was included because it has been suggested that it plays a secondary role in the reconnection intensity by affecting the efficiency.

Despite these physical motivations, the last three parameters, $E_{\mathrm{yz}}, p_{\mathrm{sw}}$, and $M_{\mathrm{A}}$ are highly correlated in the solar wind. They all depend on the solar wind velocity. Both $E_{\mathrm{yz}}$ and $M_{\mathrm{A}}$ depend on the IMF (though $E_{\mathrm{yz}}$ is only dependent on two components of the magnetic field), and both $p_{\mathrm{sw}}$ and $M_{\mathrm{A}}$ depend on the solar wind density. It is therefore possible that in subtracting off the dependence of $I_{\text {tot }}$ on $E_{\mathrm{yz}}$ and then the dependence on $p_{\text {sw }}$ we inadvertently removed a stronger dependence on $M_{\mathrm{A}}$ giving a false impression of a weak influence of $M_{\mathrm{A}}$.

To test for the possibility that our analysis has masked different dependencies than obtained above, we also used multivariable regression to determine $I_{\text {tot }}$ as a function of all three parameters simultaneously. For each clock angle bin we fit $I_{\text {tot }}$ to the following:

$F\left(E_{\mathrm{yz}}, p_{\mathrm{sw}}, M_{\mathrm{A}}\right)=m_{0}+m_{1} E_{\mathrm{yz}}+m_{2} p_{\mathrm{sw}}+m_{3} M_{\mathrm{A}}$

using the formalism described in Korth et al. (2004a), which also yields estimates for the uncertainties in the $m_{i}$. The results are given in Table 3. Comparing these results with those given above in Table 1 we find broad agreement: the $E_{\mathrm{yz}}$ and $p_{\text {sw }}$ coefficients' $1-\sigma$ ranges overlap in all cases except the $315^{\circ}$ clock angle bin, and the $M_{\mathrm{A}}$ coefficients are the smallest by a significant margin and in many cases the 1- $\sigma$ range is consistent with zero. Thus, we obtain the same $E_{\mathrm{yz}}$ and $p_{\text {sw }}$ dependencies and get the same result that the influence of $M_{\mathrm{A}}$ is weak, possibly even consistent with no statistically significant variation of $I_{\text {tot }}$ with $M_{\mathrm{A}}$.
Table 3. Correlation coefficients between total Birkeland current, $I_{\text {tot }}$, and solar wind electric field, $E_{\mathrm{yz}}$, solar wind ram pressure, $p_{\mathrm{sw}}$, and solar wind Alfvén Mach number, $M_{\mathrm{A}}$, determined using simultaneous multiple linear regression.

\begin{tabular}{cccc}
\hline $\begin{array}{c}\text { Clock angle } \\
{\left[{ }^{\circ}\right]}\end{array}$ & $\begin{array}{c}I_{\text {tot }} / E_{\mathrm{yz}} \\
{\left[\mathrm{MA} /\left(\mathrm{mVm}^{-1}\right)\right]}\end{array}$ & $\begin{array}{c}I_{\text {tot }} / p_{\mathrm{sw}} \\
{[\mathrm{MA} / \mathrm{nPa}]}\end{array}$ & $\begin{array}{c}I_{\text {tot }} / M_{\mathrm{A}} \\
{\left[\mathrm{MA} / \mathrm{unitM}_{\mathrm{A}}\right]}\end{array}$ \\
\hline 0 & $-0.03 \pm 0.10$ & $0.25 \pm 0.05$ & $0.04 \pm 0.04$ \\
45 & $0.03 \pm 0.05$ & $0.05 \pm 0.04$ & $0.00 \pm 0.02$ \\
90 & $0.41 \pm 0.07$ & $0.13 \pm 0.05$ & $0.08 \pm 0.03$ \\
135 & $0.64 \pm 0.08$ & $0.25 \pm 0.07$ & $-0.00 \pm 0.03$ \\
180 & $0.67 \pm 0.07$ & $0.39 \pm 0.06$ & $-0.05 \pm 0.02$ \\
225 & $0.68 \pm 0.09$ & $0.12 \pm 0.05$ & $0.02 \pm 0.03$ \\
270 & $-0.02 \pm 0.12$ & $0.24 \pm 0.07$ & $-0.04 \pm 0.03$ \\
315 & $0.10 \pm 0.04$ & $0.33 \pm 0.04$ & $-0.03 \pm 0.01$ \\
\hline
\end{tabular}

\section{Discussion}

Statistical analysis of Birkeland current distributions derived from magnetic field observations by the constellation of Iridium satellites shows that the large-scale Birkeland currents are controlled most strongly by the IMF orientation and the solar wind electric field. The solar wind ram pressure exerts a secondary influence, and we found no effect from the solar wind Alfvén Mach number. The correspondence between the Birkeland current patterns and the expected ionospheric projection of reconnection flows (P1) suggests that magnetopause reconnection is the dominant mechanism exercising control over the Birkeland currents. The strongest variation with solar wind conditions within a given IMF clock angle range is due to the solar wind electric field, $E_{\mathrm{yz}}$.

Magnetic reconnection taps a fraction of the solar wind potential, providing the magnetospheric convection potential, $\phi_{\mathrm{m}}$, which is impressed on the ionosphere via equipotential magnetic field lines (e.g., Reiff et al., 1981). For nominal, that is not extreme, solar wind conditions considered in this study, the transpolar cap potential, $\phi_{\mathrm{pc}}$, is assumed to equal $\phi_{\mathrm{m}}$ so that the Birkeland current intensity is proportional to $\phi_{\mathrm{m}}$. This assumption is not valid during active times when saturation effects come into play (Hill et al., 1976). In the non-saturated regime, magnetic reconnection implies that the Birkeland current density, $j_{\mathrm{r}}$, is related to drivers in the solar wind by (Siscoe et al., 2002a,b):

$j_{\mathrm{r}} \sim \phi_{\mathrm{m}} \sim E_{\mathrm{sw}} p_{\mathrm{sw}}^{-1 / 6} f_{\mathrm{r}} F(\alpha)$,

where $E_{\mathrm{sw}}$ and $p_{\mathrm{sw}}$ are the solar wind electric field and dynamic pressure, respectively, and $f_{\mathrm{r}}$ is the reconnection efficiency. The function $F(\alpha)$, where $F\left(0^{\circ}\right)=0$ and $F\left(180^{\circ}\right)=$ 1 , represents the IMF clock angle dependence of magnetopause reconnection. For constant conditions at the ionosphere, Eq. (7) implies that an increase in reconnection efficiency yields an intensification of the magnetospheric convection potential and, hence, the Birkeland current density. 

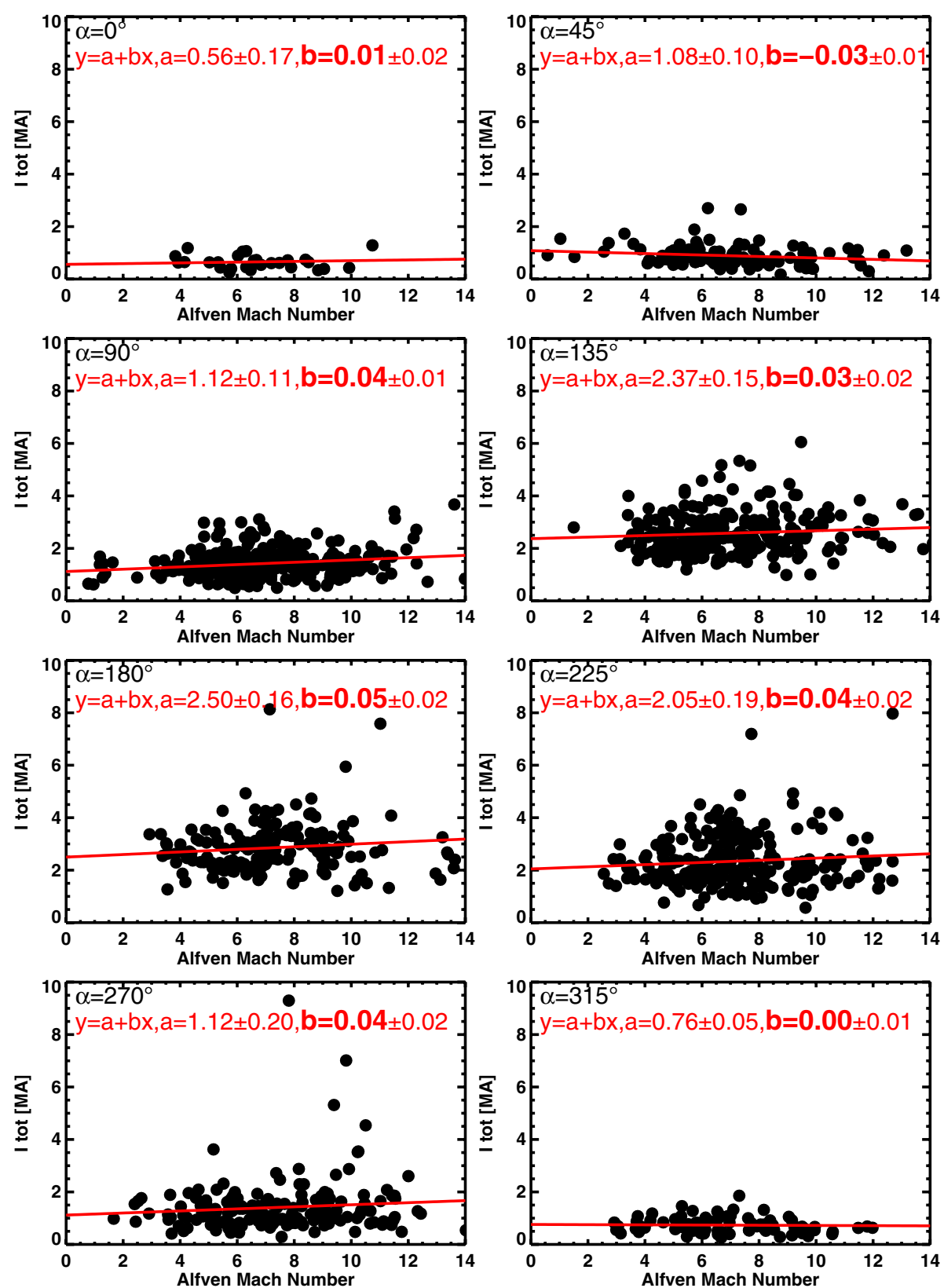

Fig. 9. Dependence of the Birkeland total current on the solar wind Alfvén Mach number, $M_{\mathrm{A}}$, and IMF clock angle, $\alpha$. The parameters and $1-\sigma$ uncertainties of the linear fits (red lines) of these data are given at the top of each panel.

The dependencies of the Birkeland currents on the solar wind electric field identified in Sect. 2.4 agree with expectations from reconnection. Figure 2 reflects the linear dependence of the total current and hence, the current densities on $E_{\mathrm{yz}}$ implied by Eq. (7) for a wide range of IMF clock angles. The dependence varies with IMF clock angle and is largest for southward IMF and weakest for northward IMF, represented by the function $F(\alpha)$ in Eq. (7). In Fig. 2, the dependence on $E_{\mathrm{yz}}$ is rather weak, the slopes for $\alpha=0^{\circ}$ and $\alpha=45^{\circ}$ are lower in magnitude than the associated 1$\sigma$ uncertainties, indicating that the intensification with $E_{\mathrm{yz}}$ is modest for northward IMF. However, we note that the current for extreme conditions and northward IMF (Korth et al., 2005; Merkin et al., 2007) is markedly larger than the events used here.

With respect to ram pressure, Eq. (7) predicts a decrease in the Birkeland current density with increasing solar wind dynamic pressure whereas the opposite is observed. Instead 
of weakly diminishing Birkeland current densities predicted by Eq. (7), the Iridium distributions show intensifications of $I_{\text {tot }}$ with increasing solar wind dynamic pressure for all IMF orientations.

An alternate means for transport of energy and momentum from the solar wind into the magnetosphere is viscous interaction between the solar wind flow and the magnetospheric circulation (Axford and Hines, 1961). The magnetospheric plasma flow, $\boldsymbol{v}$, driven by viscous interaction generates currents, $J_{\perp}$, perpendicular to the magnetic lines of force, $\boldsymbol{B}$, and field-aligned Birkeland currents, $J_{\|}$, arise from the requirement for current continuity (e.g., Parks, 2004):

$\nabla \cdot \boldsymbol{J}_{\|}=-\nabla \cdot \boldsymbol{J}_{\perp}=-\frac{\boldsymbol{B} \times \nabla p}{B^{2}}-\rho \frac{\boldsymbol{B}}{B^{2}} \times(\boldsymbol{v} \cdot \nabla) \boldsymbol{v}$.

In Eq. (8), $p$ and $\rho$ are the plasma pressure and mass density, respectively. The viscous dynamo is believed to be primarily driven by the Kelvin-Helmholtz instability caused by shear flows between the solar wind and magnetospheric plasmas at the magnetopause (Rostoker et al., 1987). For a tangential discontinuity in an incompressible magnetized plasma, the condition for instability of a wave propagating in direction $\hat{\boldsymbol{k}}$ is given by (e.g., Landau and Lifschitz, 1960; Kivelson and Chen, 1995):

$$
\left[\hat{\boldsymbol{k}} \cdot\left(\boldsymbol{v}_{2}-\boldsymbol{v}_{1}\right)\right]^{2}>\frac{\rho_{1}+\rho_{2}}{\mu_{0} \rho_{1} \rho_{2}}\left[\left(\boldsymbol{B}_{1} \cdot \hat{\boldsymbol{k}}\right)^{2}+\left(\boldsymbol{B}_{2} \cdot \hat{\boldsymbol{k}}\right)^{2}\right],
$$

where $\mu_{0}$ is the vacuum permeability and the indices 1 and 2 represent the two sides of the discontinuity. Assuming that the Kelvin-Helmholtz waves travel anti-sunward, the instability condition Eq. (9) is most readily satisfied for purely northward or southward IMF direction, for which the draped magnetosheath field at the flanks has no component in the flow direction. The viscous interaction thus might be expected to be favored under these conditions leading to a stronger dependence of the Birkeland currents on solar wind conditions. This expectation is consistent with the statistical analysis shown in Fig. 8. The Iridium observations yield persistent positive correlations between the Birkeland total current and $p_{\text {sw }}$ as one would anticipate if the field-aligned current intensity is modulated by the solar wind flow velocity for a fixed electric field. (Recall that the $E_{\mathrm{yz}}$ dependence was removed in the pressure analysis.) Furthermore, the Iridium observations exhibit local minima for dawnward and duskward IMF in the influence of ram pressure on the Birkeland total current consistent with Eq. (9). This indicates that the viscous interaction drives at least a portion of the Birkeland currents.

Finally, we consider the implications of the lack of significant influence of the solar wind Alfvén Mach number. The reconnection efficiency $f_{\mathrm{r}}$ in Eq. (7) is defined as the ratio of the inflow velocity from the magnetosheath into the reconnection line, $v_{\mathrm{r}}$, and the Alfvén velocity, $v_{\mathrm{A}}$ (Petschek, 1964). Determining the value of $f_{\mathrm{r}}$ is difficult because it depends on assumed boundary conditions and on the locations where $v_{\mathrm{r}}$ and $v_{\mathrm{A}}$ are measured (Sonnerup, 1974). Furthermore, Sonnerup (1970) argued that reconnection should be entirely absent in high- $\beta$ plasmas, where $\beta=2 \mu_{0} p / B^{2}$ is the ratio of gas pressure to magnetic pressure. Indeed, previous observations consistently link increases in the plasma $\beta$ to decreased reconnection efficiency (Paschmann et al., 1986; Scurry et al., 1984; Phan et al., 1996). This finding was independently confirmed by Anderson et al. (1997) from comparison of AMPTE/IRM and AMPTE/CCE observations of plasma depletion in the subsolar quasi-perpendicular magnetosheath. Because the geocentric distance for apogee of these spacecraft, $8.8 R_{\mathrm{E}}$ for CCE and $18.8 R_{\mathrm{E}}$ for IRM, differ significantly, the spacecraft encounter the magnetosheath for distinct ranges in the solar wind Alfvén Mach number. From the relative difference in the reconnection efficiency identified in the two sets of observations, Anderson et al. (1997) inferred $f_{\mathrm{r}} \sim 1 / \beta_{\mathrm{sh}}$ for $\beta_{\mathrm{sh}}>1$ measured in the magnetosheath. Assuming a quasi-perpendicular bow shock in the strong shock limit for which $\beta_{\text {sh }}=3 / 32 M_{\text {Asw }}^{2}$, these authors further correlate $f_{\mathrm{r}}$ with the solar wind Alfvén Mach number, $M_{\text {Asw }}$ :

$$
f_{\mathrm{r}} \sim \frac{1}{\beta_{\mathrm{sh}}} \sim \frac{1}{M_{\mathrm{Asw}}^{2}} .
$$

Relation (10) predicts the reconnection efficiency and, therefore, the Birkeland total current to be strongly anti-correlated with solar wind Alfvén Mach number. Instead, the statistical analysis presented in Fig. 9 showed, if anything, a slight positive correlation of the current with $M_{\text {Asw }}$. The analysis presented here is for modest forcing, i.e., low $M_{\mathrm{Asw}}$ and $\beta_{\mathrm{sh}}$, such that the reconnection efficiency changes are not evident for the range of forcing represented by this data set. Examination of extreme conditions may be needed to reflect the influence of decreasing reconnection efficiency, but it is not a determining factor for the nominal ranges of forcing considered here. It may be that the decreases in reconnection efficiency do not reduce the solar wind dynamo potential imposed on the M-I system so long as the reconnection flows can accommodate the imposed solar wind flow. Only when the imposed solar wind flow exceeds the flow that reconnection can accommodate will the variation in reconnection efficiency be evident. We therefore conclude that for nominal conditions, the reconnection efficiency is more than sufficient to take up the imposed solar wind flow. The decrease in reconnection efficiency is therefore an effect that does not come into play for the range of solar wind forcing considered here.

In addition to modulating the current intensity, the solar wind electric field and the solar wind dynamic pressure modify the current patterns. With increasing $E_{\mathrm{yz}}$ the currents expand equatorward and shift toward noon. The expansion of the Birkeland currents to lower latitudes indicates expansion of the polar cap and hence an increase in the open magnetic flux. The amount of open flux is controlled by the relative reconnection rates at the dayside magnetopause and in 
the magnetotail (Siscoe and Huang, 1985). When the solar wind electric field increases, so does the reconnection rate at the dayside magnetopause while that in the magnetotail remains initially unchanged. The polar cap then responds to the increase in open magnetic flux by expanding equatorward. Since Region-1 Birkeland currents are intimately tied to the location of the boundary between open and closed magnetic flux, the currents must follow the expansion to lower latitudes as well. Similarly, the Birkeland currents retract poleward when the solar wind electric field decreases and the dayside reconnection rate decreases relative to that in the magnetotail, diminishing the amount of lobe magnetic flux. Finally, the equatorward expansion of the Birkeland currents with increasing $p_{\mathrm{sw}}$ is consistent with the solar wind ram pressure modulating the size of the magnetosphere. An increase in $p_{\text {sw }}$ compresses the magnetic field inside the magnetosphere, and the ionospheric foot point of the magnetic lines of force along which the Birkeland currents flow move to lower latitudes. As the solar wind dynamic pressure decreases the magnetic field lines and the currents retreat poleward.

We suggest that the shift in the currents toward noon with increasing $E_{\mathrm{yz}}$ is also a direct consequence of the solar wind electric field controlling the magnetopause reconnecting rate. For intervals when the reconnection rate at the dayside magnetopause is enhanced compared to that in the magnetotail, more open field lines are generated, which are subsequently swept into the tail by the solar wind. The process essentially leads to an erosion of the magnetopause (Aubry et al., 1970). As the flux content of the tail increases, the cusp corresponding to newly opened field lines, moves equatorward (e.g., Newell et al., 1989). The cusp location is representative for the latitudinal extent of the ionospheric convection, implying that the convection pattern shifts to lower latitudes on the dayside. Since the large-scale Birkeland currents are tightly coupled to the convection pattern, the currents appear shifted toward noon for enhanced solar wind electric fields.

Interestingly, a shift of the large-scale Birkeland currents in the dawn-dusk direction was not observed in the present study. Anderson et al. (2005) identified a dawn-dusk asymmetry in the large-scale Birkeland currents during geomagnetic storms. Therefore, one might expect to find a dawndusk asymmetry in the statistical current distributions for elevated $E_{\mathrm{yz}}$ but this is not the case. Anderson et al. (2005) attributed the storm-time dawn-dusk asymmetry in the currents to the partial ring current. The partial ring current is most pronounced during the storm main phase. It is likely that the number of storm-time intervals included in the present analysis is insufficient to detect the dawn-dusk asymmetry, first because storm main-phase currents tend not to be stable and second because the range of electric fields with good statistics in this study are considerably lower than storm-time conditions. The quiet to moderate $E_{\mathrm{yz}}$ range included in this study may simply not represent the storm-time time conditions examined by Anderson et al. (2005). That no systematic dawn-dusk asymmetry is identified for low to moderate
$E_{\mathrm{yz}}$ indicates that the storm-time magnetosphere may not be simply represented by an extrapolation of low-to-medium activity conditions.

\section{Summary and conclusions}

We have examined the dependence of the large-scale Birkeland currents on IMF and solar wind parameters. That the IMF orientation most fundamentally determines the distribution of the Birkeland currents was already discussed in P1. Here we have extended the analysis to dependencies on solar wind electric field, dynamic pressure, and Alfvén Mach number. The key findings are:

1. The strength of the solar wind electric field, $E_{\mathrm{yz}}$, is the dominant factor controlling the total current $\left(0.8 \mathrm{MA} /\left(\mathrm{mV} \mathrm{m}^{-1}\right)\right.$ for purely southward IMF);

2. The solar wind electric field strength also affects the distribution of currents in two ways: (1) Increasing $E_{\mathrm{yz}}$ causes an equatorward expansion by $5.5 \% /\left(\mathrm{mV} \mathrm{m}^{-1}\right)$ for southward IMF and less, $2.5 \% /\left(\mathrm{mV} \mathrm{m}^{-1}\right)$, for $|B y / B z| \approx 1$; (2) increasing $E_{\mathrm{yz}}$ also shifts the currents toward noon by $0.4^{\circ} /\left(\mathrm{mVm}^{-1}\right)$;

3. Normalizing the Birkeland currents to the median solar wind electric field, the solar wind dynamic pressure leads to an increase in the total current by $0.4 \mathrm{MA} / \mathrm{nPa}$ for purely southward IMF and less, $\sim 0.2 \mathrm{MA} / \mathrm{nPa}$, for other IMF orientations;

4. Normalizing the Birkeland currents to both the median solar wind electric field and dynamic pressure, there is no detectable dependence of the total current on solar wind Alfvén Mach number. This implies that subsolar reconnection can accommodate the range of flows imposed by the solar wind dynamo under typical (nonextreme) conditions. This is consistent with the appearance of a subsolar plasma depletion layer only under extreme solar wind pressures and electric fields (cf. Anderson et al., 1997).

The quantitative relationships between characteristic solar wind parameters and the Birkeland current distribution and intensity can be used to test physical models of the magnetosphere-ionosphere system. These comparisons will allow us to constrain the physical processes governing the system's dynamics.

Acknowledgements. We thank Iridium Communications Inc. for providing the engineering magnetometer data for scientific analysis. We thank the ACE team for the use of the MAG and SWEPAM data made available via the ACE Level 2 data base. Support for processing and analysis of the Iridium magnetometer data was provided by NSF under grant ATM-0539024. Any opinions, findings, and conclusions or recommendations expressed in this material are those of the authors and do not necessarily reflect the views of the 
National Science Foundation.

Topical Editor K. Kauristie thanks V. P. Papitashvili and another anonymous referee for their help in evaluating this paper.

\section{References}

Amm, O.: The elementary current method for calculating ionospheric current systems from multisatellite and ground magnetometer data, J. Geophys. Res., 106, 24843-24855, 2001.

Anderson, B. J. and Korth, H.: Saturation of global field aligned currents observed during storms by the Iridium satellite constellation, J. Atmos. Solar-Terr. Phys., 69, 166-169, doi:10.1016/j. jastp.2006.06.013, 2007.

Anderson, B. J., Phan, T. D., and Fuselier, S. A.: Relationships between plasma depletion and subsolar reconnection, J. Geophys. Res., 102, 9531-9542, 1997.

Anderson, B. J., Takahashi, K., and Toth, B. A.: Sensing global Birkeland currents with Iridium engineering magnetometer data, Geophys. Res. Lett., 27, 4045-4048, 2000.

Anderson, B. J., Ohtani, S.-I., Korth, H., and Ukhorskiy, A.: Storm time dawn-dusk asymmetry of the large-scale Birkeland currents, J. Geophys. Res., 110, A12220, doi:10.1029/ 2005JA011246, 2005.

Anderson, B. J., Korth, H., Waters, C. L., Green, D. L., and Stauning, P.: Statistical Birkeland current distributions from magnetic field observations by the Iridium constellation, Ann. Geophys., 26, 671-687, 2008,

http://www.ann-geophys.net/26/671/2008/.

Aubry, M. P., Russell, C. T., and Kivelson, M. G.: Inward motion of the magnetopause before a substorm, J. Geophys. Res., 75, 7018-7031, 1970.

Axford, W. I. and Hines, C. O.: A Unifying Theory of HighLatitude Geophysical Phenomena and Geomagnetic Storms, Can. J. Phys., 39, 1443-1464, 1961.

Baker, K. B. and Wing, S.: A new magnetic coordinate system for conjugate studies at high latitudes, J. Geophys. Res., 94, 91399143, 1989.

Borovsky, J. E., Thomsen, M. F., and Elphic, R. C.: The driving of the plasma sheet by the solar wind, J. Geophys. Res., 103, 17617-17639, 1998.

Germany, G. A., Torr, D. G., Richards, P. G., Torr, M. R., and John, S.: Determination of Ionospheric Conductivities from FUV Auroral Emissions, J. Geophys. Res., 99, 23297-23305, 1989.

Green, D. L., Waters, C. L., Korth, H., and Anderson, B. J.: Validation of Southern Hemisphere Field-Aligned Currents Calculated from Iridium Magnetic Field Data, in: Australian Space Science Conference Series, edited by: Short, W. and Cairns, I., National Space Society of Australia Ltd, GPO Box 7048 Sydney NSW 2001, 2008.

Green, D. L., Waters, C. L., Anderson, B. J., and Korth, H.: Seasonal and interplanetary magnetic field dependence of the fieldaligned currents for both Northern and Southern Hemispheres, Ann. Geophys., 27, 1701-1715, 2009, http://www.ann-geophys.net/27/1701/2009/.

Hardy, D. A., Gussenhoven, M. S., Raistrick, R., and McNeil, W. J.: Statistical and Functional Representations of the Pattern of Auroral Energy Flux, Number Flux, and Conductivity, J. Geophys. Res., 92, 12275-12294, 1987.
Hardy, D. A., Gussenhoven, M. S., and Brautigam, D.: A Statistical Model of Auroral Ion Precipitation, J. Geophys. Res., 94, 370392, 1989.

Hill, T. W., Dessler, A. J., and Wolf, R. A.: Mercury and Mars - Role of Ionospheric Conductivity in Acceleration of Magnetospheric Particles, Geophys. Res. Lett., 3, 429-432, 1976.

Iijima, T. and Potemra, T. A.: The Relationship between InterPlanetary Quantities and Birkeland Current Densities, Geophys. Res. Lett., 9, 442-445, 1982.

Kivelson, M. G. and Chen, S.-H.: The Magnetopause: Surface Waves and Instabilities and Their Possible Dynamical Consequences, in: Physics of the Magnetopause, edited by: Song, P., Sonnerup, B. U. Ö., and Thomsen, M. F., Geophysical Monograph 90, pp. 257-268, American Geophysical Union, Washington, 1995.

Korth, H., Anderson, B. J., Acuna, M. H., Slavin, J. A., Tsyganenko, N. A., Solomon, S. C., and McNutt, R. L.: Determination of the properties of Mercury's magnetic field by the MESSENGER mission, Planet. Space Sci., 52, 733-746, 2004a.

Korth, H., Anderson, B. J., Wiltberger, M. J., Lyon, J. G., and Anderson, P. C.: Intercomparison of Ionospheric Electrodynamics From the Iridium Constellation With Global MHD Simulations, J. Geophys. Res., 109, A07307, doi:10.1029/2004JA010428, 2004b.

Korth, H., Anderson, B. J., Frey, H. U., and Waters, C. L.: Highlatitude electromagnetic and particle energy flux during an event with sustained strongly northward IMF, Ann. Geophys., 23, 1295-1310, 2005, http://www.ann-geophys.net/23/1295/2005/.

Korth, H., Anderson, B. J., Ruohoniemi, J. M., Frey, H. U., Waters, C. L., Immel, T. J., and Green, D. L.: Global observations of electromagnetic and particle energy flux for an event during northern winter with southward interplanetary magnetic field, Ann. Geophys., 26, 1415-1430, 2008, http://www.ann-geophys.net/26/1415/2008/.

Landau, L. D. and Lifschitz, E. M.: Electrodynamics of Continous Media, Pergamon Press, New York, 1960.

McComas, D. J., Bame, S. J., Barker, P. L., Feldman, W. C., Phillips, J. L., Riley, P., and Griffee, J. W.: Solar Wind Electron Proton Alpha Monitor (SWEPAM) for the Advanced Composition Explorer, Space Sci. Rev., 86, 563-612, 1998.

Merkin, V. G., Lyon, J. G., Anderson, B. J., Korth, H., Goodrich, C. C., and Papadopoulos, K.: A global MHD simulation of an event with a quasi-steady northward IMF component, Ann. Geophys., 25, 1345-1358, 2007, http://www.ann-geophys.net/25/1345/2007/.

Newell, P. T., Meng, C.-I., and Sibeck, D. G.: Some low-altitude cusp dependencies on the interplanetary magnetic field, J. Geophys. Res., 94, 8921-8927, 1989.

Ober, D. M., Maynard, N. C., and Burke, W. J.: Testing the Hill model of transpolar potential saturation, J. Geophys. Res., 108, 1467, doi:10.1029/2003JA010154, 2003.

Papitashvili, V. O., Christiansen, F., and Neubert, T.: A new model of field-aligned currents derived from high-precision satellite magnetic field data, Geophys. Res. Lett., 29, 1683, doi:10.1029/ 2001GL014207, 2002.

Parks, G. K.: Physics of Space Plasmas, Westview Press, Boulder, 2nd edn., 2004.

Paschmann, G., Papamastorakis, I., Baumjohann, W., Sckopke, N., 
Carlson, C. W., Sonnerup, B. U. Ö., and Lühr, H.: The Magnetopause for Large Magnetic Shear - AMPTE/IRM Observations, J. Geophys. Res., 91, 11099-11115, 1986.

Petschek, H. E.: Magnetic field annihilation, The Physics of Solar Flares, NASA Spec. Publ., 50, 425-439, 1964.

Phan, T. D., Paschmann, G., and Sonnerup, B. U. Ö.: Low-latitude dayside magnetopause and boundary layer for high magnetic shear, 2, Occurrence of magnetic reconnection, J. Geophys. Res., 101, 7817-7828, 1996.

Potemra, T. A., Zanetti, L. J., Bythrow, P. F., Lui, A. T. Y., and Iijima, T.: $B_{\mathrm{y}}$-Dependent Convection Patterns During Northward IMF, J. Geophys. Res., 89, 9753-9760, 1984

Rasmussen, C. E., Schunk, R. W., and Wickwar, V. B.: A Photochemical Equilibrium Model for Ionospheric Conductivity, J. Geophys. Res., 93, 9831-9840, 1988.

Reiff, P. H., Spiro, R. W., and Hill, T. W.: Dependence of Polar Cap Potential Drop on Interplanetary Parameters, J. Geophys. Res., 86, 7639-7648, 1981.

Rostoker, G., Baker, D. N., Lemaire, J., and Vasyliunas, V.: Dialog on the Relative Roles of Reconnection and the "Viscous" Interaction in Providing Solar-Wind Energy to the Magnetosphere, In Magnetotail Physics (A. T. Y. Lui, Ed.), pp. 257-268, Johns Hopkins Univ. Press, Baltimore, 1987.

Scurry, L., Russell, C. T., and Gosling, J. T.: Geomagnetic-Activity and the Beta-Dependence of the Dayside Reconnection Rate, J. Geophys. Res., 99, 14811-14814, 1984.

Siscoe, G. L. and Huang, T. S.: Polar cap inflation and deflation, J. Geophys. Res., 90, 543-547, 1985

Siscoe, G. L., Crooker, N. U., and Siebert, K. D.: Transpolar potential saturation: Roles of region 1 current system and solar wind ram pressure, J. Geophys. Res., 107, 1321, doi:10.1029/ 2001JA009176, 2002a.

Siscoe, G. L., Erickson, G. M., Sonnerup, B. U. Ö., Maynard, N. C., Schoendorf, J. A., Siebert, K. D., Weimer, D. R., White, W. W., and Wilson, G. R.: Hill model of transpolar potential saturation: Comparisons with MHD simulations, J. Geophys. Res., 107, 1075, doi:10.1029/2001JA000109, 2002b.
Smith, C. W., L'Heureux, J., Ness, N. F., Acuña, M. H., Burlaga, L. F., and Scheifele, J.: The ACE Magnetic Fields Experiment, Space Sci. Rev., 86, 613-632, 1998.

Sonnerup, B. U. Ö.: Magnetic field reconnection in a highly conducting incompressible fluid, J. Plasma Phys., 4, 161-174, 1970.

Sonnerup, B. U. Ö.: Magnetopause Reconnectin Rate, J. Geophys. Res., 79, 1546-1549, 1974.

Sotirelis, T. and Meng, C. I.: Magnetopause from pressure balance, J. Geophys. Res., 104, 6889-6898, 1999.

Waters, C. L., Anderson, B. J., and Liou, K.: Estimation of global field aligned currents using the Iridium System magnetometer data, Geophys. Res. Lett., 28, 2165-2168, 2001.

Weimer, D. R.: Maps of ionospheric field-aligned currents as a function of the interplanetary magnetic field derived from Dynamics Explorer 2 data, J. Geophys. Res., 106, 12889-12902, 2001.

Weimer, D. R.: Improved ionospheric electrodynamic models and application to calculating Joule heating rates, J. Geophys. Res., 110, A05306, doi:10.1029/2004JA010884, 2005.

Wing, S. and Newell, P. T.: Central plasma sheet ion properties as inferred from ionospheric observations, J. Geophys. Res., 103, 6785-6800, 1998

Zanetti, L. J., Potemra, T. A., Iijima, T., Baumjohann, W., and Bythrow, P. F.: Ionospheric and Birkeland Current Distributions for Northward Interplanetary Magnetic Field: Inferred Polar Convection, J. Geophys. Res., 89, 7453-7458, 1984.

Zhang, Y. and Paxton, L. J.: An empirical Kp-dependent global auroral model based on TIME/GUVI FUV data, J. Atmos. SolarTerr. Phys., 79, 1231-1242, doi:10.1016/j.jastp.2008.03.008, 2008 . 